Hammersmith Hospital, and Institutes of Neurology and Child Health, London, UK. Two patients had abetalipoproteinemia and vitamin E was undetectable from birth. One had a familial vitamin E defiency, and had been diagnosed as Friedreich's ataxia at 13 years of age. One had Crohn's disease and fat malabsorption dating back to 16 years. Three had striking head tremor. Impaired nigrostriatal activity in the patients with abetalipoproteinemia, demonstrated by reduced dopa uptake using PET studies, was similar to that seen in Parkinson's disease. (Dexter DT et al. Nigrostriatal function in vitamin E deficiency: Clinical, experimental, and positron emission tomographic studies. Ann Neurol March 1994; $\underline{35}$ :298-303). (Respond: Prof AE Harding, University Dept of Clinical Neurology, Institute of Neurology, Queen Square, London, WC1N 3BG, UK).

COMMENT. Some children with typical signs of Friedreich's ataxia have familial vitamin E deficiency syndrome, with autosomal recessive inheritance. (Ped Neur Briefs Dec 1993; $\underline{7}: 91$ ). Early identification and supplementation with vitamin $\mathrm{E}$ may halt progression of the ataxia. A dose of $800 \mathrm{mg} /$ day vitamin $\mathrm{E}$ in the 43-year-old male patient with this syndrome reported above had stabilized the neurologic status and his serum level of vitamin E was normal.

\title{
CEREBELLAR ATAXIA AND CSF FOLATE DEFICIENCY
}

A slowly progressive cerebellar syndrome associated with disturbed folate transfer across the choroid plexus is reported in an 18-year-old male who presented with incoordination of hands and feet at the Institute of Neurology, University Hospital of Nijmegen, The Netherlands. A rapidly progressive bilateral sensorineural hearing loss had preceeded the onset of ataxia which was complicated by dysarthria and dysphagia, and was followed at 21 years, with muscle cramps and at 26 years, with a distal spinal muscular atrophy and pyramidal tract signs of hyperreflexia and Babinski reflexes. Cranial CT showed cerebral and cerebellar atrophy and hypodensities in the basal ganglia. The serum $(9.6-10.5 \mathrm{nmol} / \mathrm{l})$ and red cell (371) folate levels were normal while the CSF folate was severely depleted (1.4-2.6 nmol/1; ref normal range 14-42). Analyses of folate binding protein in CSF performed at laboratories in Denmark showed abnormalities that indicate a defective folate transport into the CNS. (Wevers RA et al. Folate deficiency in cerebrospinal fluid associated with a defect in folate binding protein in the central nervous system. I Neurol Neurosurg Psychiatry Feb 1994;57:223-226). (Respond: Dr RA Wevers, Institute of Neurology, University of Nijmegen, PO Box 9101, 6500 HB Nijmegen, The Netherlands).

COMMENT. Folate occurs in higher concentrations in CSF than in plasma, and it enters the CSF against a concentration gradient. Folate binding proteins in the plasma membrane of the choroid plexus are essential in the transport of folate to the CSF and CNS. Low CSF folate has been reported in inborn errors of metabolism, Kearns-Sayre syndrome, and HIV infection. Neurologic manifestations of inherited disorders of folate metabolism include mental and motor retardation, ataxia, and seizures. Consanguinity of the parents of the above patient suggests an autosomal recessive inheritance.

In addition to folate and vitamin E deficiencies, other degenerative ataxias resembling Friedreich's ataxia that may be amenable to dietary supplements or modifications include vitamin $\mathrm{B}_{12}$ and biotin deficiencies and Refsum's disease, responsive to a diet low in 
phytol and phytanic acid.(Progress in Pediatric Neurology, Chicago, PNB Publ, 1991, p 480).

\section{CEREBELLAR ATAXIA AND MULTIPLE SCLEROSIS}

Clinical manifestations and MRI findings in four Japanese children with multiple sclerosis are reported from the Department of Pediatrics, Sapporo Medical University, Japan. Three presented with gait ataxia and one developed cerebellar intention tremor within 2 months of an onset with weakness of the right arm and speech impairment. The age of onset of symptoms was at 7-12 years. All had optic neuritis. MRI showed multiple white matter lesions and demyelinating plaques in cerebral hemispheres, cerebellum and brain stem. CT abnormalities were indefinite or absent.(Wakai $S$ et al. Childhood multiple sclerosis: MR images and clinical variations in four Japanese cases. Brain Dev 1994; $16: 52-56$ ). (Respond: Dr S Wakai, Dept of Pediatrics, School of Medicine, Sapporo Medical University, South 1 West 16, Chuo-ku, Sapporo 060, Japan).

COMMENT. MRI was more sensitive than CT in diagnosis of demyelination in these patients. One of the 4 children had presented with Devic disease and 2 years later developed chronic inflammatory demyelinating polyradiculoneuropathy. A survey of 55 pediatric patients with MS in Japan by Prof Y Fukuyama and associates (1991) had found peripheral nerve involvement in $10(17 \%)$.

\section{TOXIC DISORDERS}

\section{COCAINE EXPOSURE IN CHILDHOOD: NEUROLOGIC SIGNS}

Neurologic manifestations of cocaine exposure in $19(46 \%)$ of 41 children, ages 2 months to 18 years, found to have cocaine-positive urine screening tests during a 1-year period (Jan - Dec 1990), are reported from the Children's National Medical Center, Washington, DC. Seizures were the most common symptom of neurotoxicity, occurring in 7 children in the age range 1-8 years. Obtundation occurred in 6 (ages 5 mos-18 yrs); delirium in 4 (ages 16-19 yrs); dizziness 1 and drooling 2 (ages 16-18 yrs). Seizure patterns were focal with secondary generalization in 3 , generalized in 4 , and were associated with fever in 2. Passive intoxication in a closet where "crack" was smoked was the most likely cause of exposure in young children. An additional 14 adolescent patients with positive urine screens had neurologic complications of head injuries, and cocaine-related symptoms could not be evaluated. (Mott $\mathrm{SH}$ et al. Neurologic manifestations of cocaine exposure in childhood. Pediatrics April 1994;93:557-560). (Reprints: Dr Stephen H Mott, Neurology Dept, Children's National Medical Center, 111 Michigan Ave, NW, Washington, DC 20010).

COMMENT. Seizures were the commonest manifestation of cocaine exposure and neurotoxicity in young children. Adolescents suffered from alterations in mental status. Urine screen for possible cocaine exposure is recommended in children with first-time seizures, afebrile or febrile.

A study of the relationship between maternal cocaine dependency and child maltreatment at Yale University School of Medicine showed that 47 infants cocaine-exposed in utero were at 